\title{
Development Mechanical Characteristic of Magneto-Rheological (MR) Fluid by Adding Graphene Oxide
}

\author{
Jinhyeok Park ${ }^{1, a}$, Soomin Kim ${ }^{1, b}$ Pyungwha Kim ${ }^{1, \mathrm{c}}$, \\ Jye Ung Jung ${ }^{1, d}$, Chang-Hyun Cho ${ }^{1, e}$, Seung-Bok Choi ${ }^{1, f}$ \\ ${ }^{1}$ Smart Structures and Systems Laboratory, Department of Mechanical Engineering, Inha \\ University, Incheon, Korea, 402-751

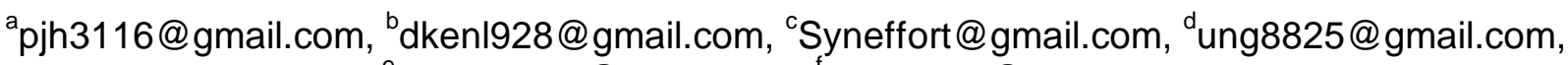 \\ echang9465@gmail.com, 'seungbok@inha.ac.kr
}

Keywords: MR Fluid. Graphene.

Abstract. In this paper, mechanical properties of magneto-rheological(MR) fluid is compared with MR fluid which is added by graphene. We mix the MR fluid with graphene oxide (GO) by $0.33 \%$ mass fractions (G33). Adding the graphene into MR fluid has an effect on the viscosity. Furthermore, MR fluid largely changed the magnetic characteristics when the magnetic field is generated. The G33 increases the shear stress on same condition due to the GO. The response time of MR fluid and G33 are similar. However shear stress of G33 is bigger than MR fluid itself and saturation time is much faster. The presented results demonstrate advantage of adding the GO into MR fluid.

\section{Introduction}

MR fluid consists of rheological materials that change rheological phase-change under the application of external magnetic fields. Generally, MR fluid are composed of soft ferromagnetic or paramagnetic particles $(0.03 \sim 10 \mu \mathrm{m})$ dispersed in a carrier fluid. A many different ceramic metal and various materials can be used in the composition of MR fluid [1]. Usually, the MR particles are pure iron, carbonyl iron, or cobalt powder and the carrier fluid which is a non-magnetic, organic, or aqueous liquid, a silicone oil. MR fluid can be shown liquid-like properties without magnetic excitation and can form chain structures when an external magnetic field is applied [2]. Because of this unique characteristic, the yield stress and response time and viscosity of MR fluid has been thoroughly researched and improved. Moreover, MR fluid has been applied to a damper, clutch and brake and other various mechanic systems [3]. Therefore the response time and shear stress of MR fluid is much important to use in the mechanical systems. Adding GO is helpful to change the mechanical characteristic, and viscosity [4]. In order to improve the shear stress and response time, we propose the particles in 140CG from Lord Corporation which are modified by adding GO.

The 140CG MR fluid of Lord Corporation and MR fluid which has GO particle mass fractions is around $33 \%$ on $140 \mathrm{CG}$. In order to identify mechanical characteristics of MR fluid added GO, we conducted diverse experimental results; time response, shear rate vs shear stress, viscosity, and magnetic intensity vs shear stress. All the experiments are conducted at room temperature $25 \mathrm{C}$. For experimental test, a computer controls a motor speed, and current signal is sent to DAQ board. DAQ board changes the digital signals to analog signals. A rod which installed motor and torque sensor rotates inside MR fluid and MR fluid forms chain structures from external magnetic flux generated from solenoid coil. Because MR fluid changes the shear stress depending on magnetic intensity, the torque sensor in rod measure the shear stress and send to DAQ board and DAQ changes the analog signal to digital signal.

\section{Experimental Result}

Time response is shown figure 1. The response time of MR fluid added GO is similar with 140CG. But, saturation time of G33 is much faster than 140CG. Though two fluids are similar with the shear stress 
without current, the average of shear stress of MR fluid added GO is much larger than 140CG when current is induced into the coil.

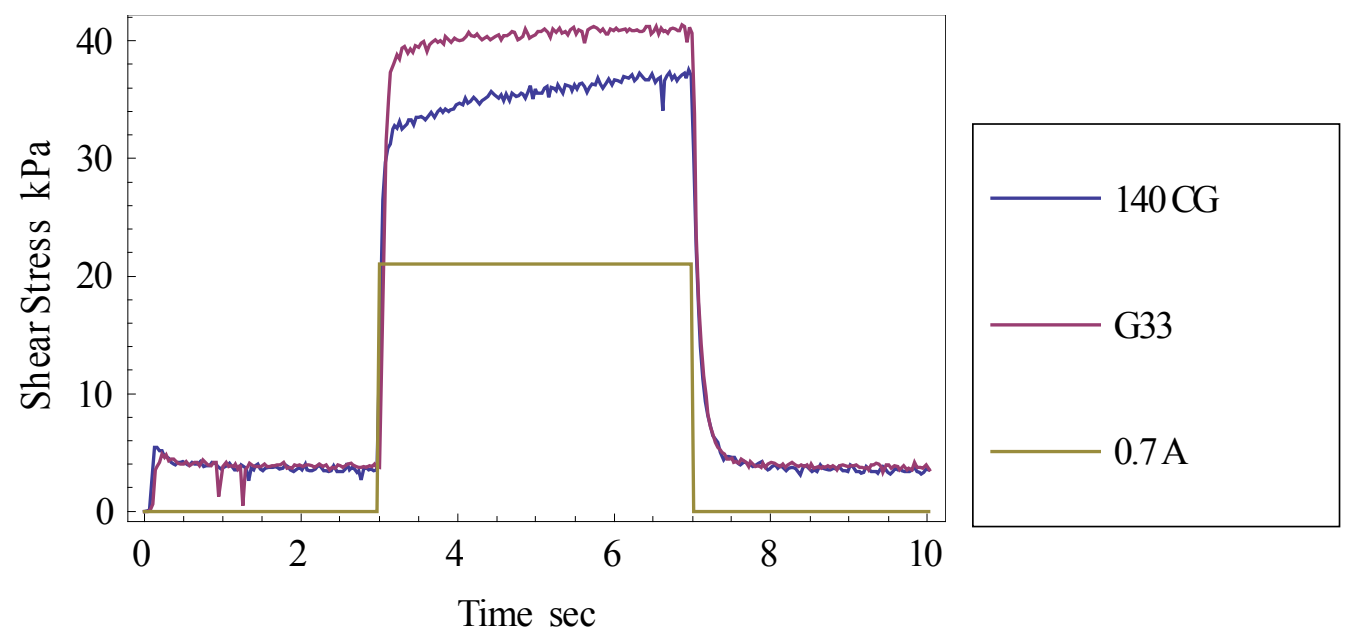

Fig. 1 Response time of magneto-rheological fluids

MR fluid has unique characteristic which can change the yield stress by induced external magnetic field. Therefore a relation between magnetic intensity $(\mathrm{H})$ and shear stress is the most important characteristic among the overall performances of MR fluid. According to Fig. 2, the shear stress sensitivity from magnetic intensity of G33 is better than 140CG.

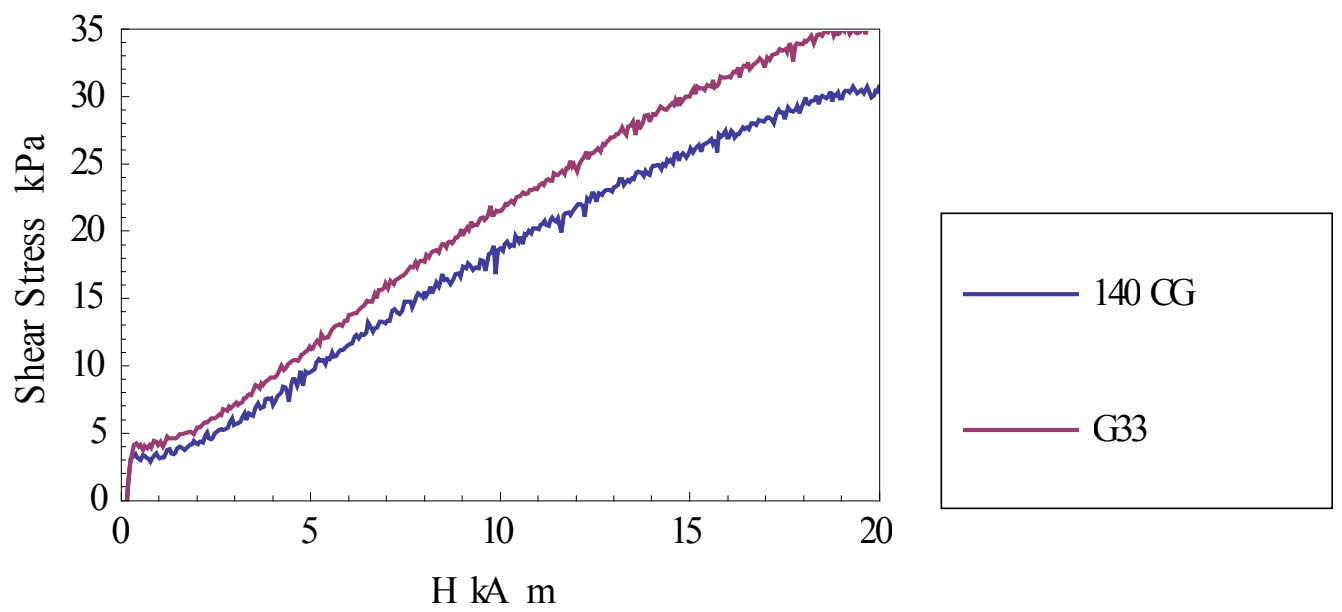

Fig. 2 Magnetic intensity $[\mathrm{kA} / \mathrm{m}]$ vs Shear stress[kPa]

However, the shear stress is bigger than before mixed without current. The viscosity is slightly increased due to the GO particles as shown in Fig. 3 


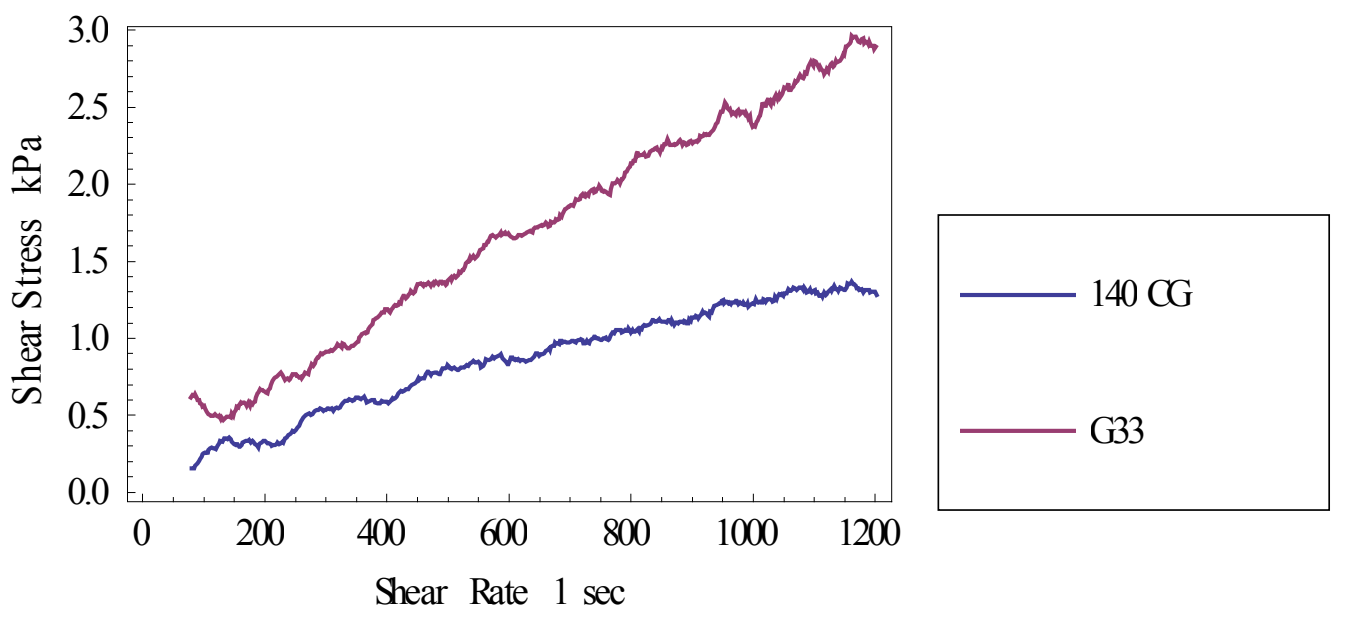

Fig. 3 Viscosity of Magneto-rheological fluids

In order to compare with the overall performance of two fluids, the shear stress is measured depending on the shear rate and magnetic field. The magnetic intensity inside the MR fluid is calculated as follows:

$$
H=\frac{N I}{2 d}
$$

where, $N$ is the number of turn of coil, $I$ is current, $\mathrm{d}$ is depth of MR fluid. According to Fig. 4, the shear stress of G33 is larger than 140CG on same condition. Furthermore, saturated shear stress is increased. On the other hand, magnetic characteristic of MR fluid is obviously improved.

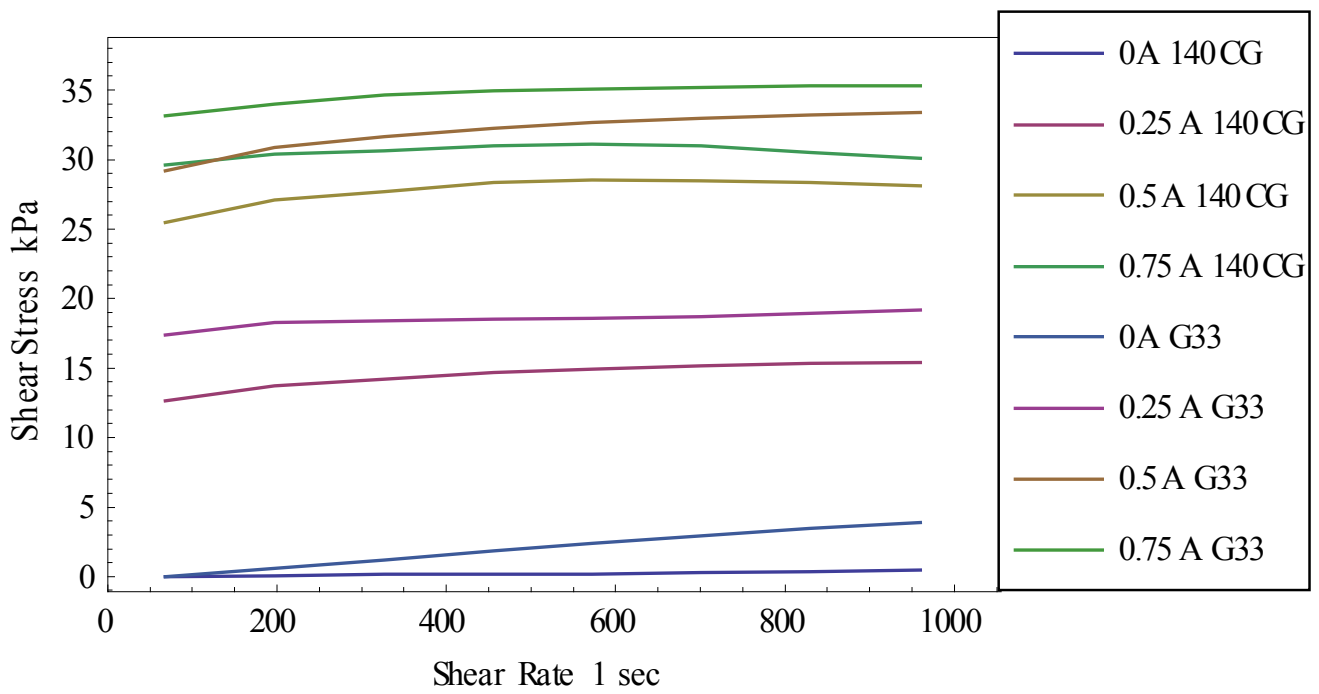

Fig. 4 Shear stress depending on shear rate and current

\section{Conclusion}

In this study, the mechanical characteristics of MR fluid were compared with MR fluid added GO. Various experiments were conducted in order to measure the time response and magnetic property change and viscosity. According to the results of experiment, although viscosity is increase due to GO, the general performance of G33 was better than the original MR fluid. These improved performances can enhance control characteristics of various applications of MR fluids such as damper, mount and clutch. 


\section{References}

[1] Choi, S. B., and Han, Y.M., in: Magnetorheological fluid technology: Applications in vehicle systems (CRC Press 2012).

[2] Jolly, M. R., Carlson, J. D., \& Munoz, B. C. A model of the behavior of magnetorheological materials., Smart Materials and Structures, Vol. 5, No.5, (1996) 607.

[3] Ha, S. H., Seong, M. S. and Choi, S. B. Design and vibration control of military vehicle suspension system using magnetorheological damper and disc spring., Smart Materials and Structures, Vol. 22, No.6, (2013) 065006.

[4] Chen, K., Zhang, W. L., Shan, L., Zhang, X., Meng, Y., Choi, H. J. and Tian, Y., Magnetorheology of suspensions based on graphene oxide coated or added carbonyl iron microspheres and sunflower oil. Journal of Applied Physics, Vol. 116,No.15, (2014), 153508. 